\title{
L'exécution des décisions de la Cour internationale de justice en matière de délimitation maritime
}

De l'existence de garanties à l'opportunité de contraintes postjuridictionnelles

The Implementation of International Courts of Justice Decisions on Maritime Delimitations

\section{Elsa Edynak}

\section{(QpenEdition Journals}

Édition électronique

URL : http://journals.openedition.org/add/801

DOI : 10.4000/add. 801

ISSN : 2606-1988

\section{Éditeur}

Presses universitaires de Rouen et du Havre

\section{Édition imprimée}

Date de publication : 1 avril 2015

Pagination : 61-92

ISSN : 1955-0855

Référence électronique

Elsa Edynak, "L'exécution des décisions de la Cour internationale de justice en matière de délimitation maritime », Les Annales de droit [En ligne], 7 | 2013, mis en ligne le 03 mai 2018, consulté le 30 avril 2019. URL : http://journals.openedition.org/add/801; DOI : 10.4000/add.801 


\title{
L'exécution des décisions de la Cour internationale de justice en matière de délimitation maritime
}

\author{
De l'existence de garanties à l'opportunité de contraintes \\ post-juridictionnelles
}

\author{
Elsa EDYNAK
}

L'espace maritime, en contraignant des États très divers à coexister, est peutêtre même à l'origine du droit international. Incités fortement à préserver l'espace maritime du chaos, les États ont peu à peu forgé un régime destiné à réaliser un juste équilibre entre les diverses formes de son utilisation (défense, commerce, pêche) et ont jeté les bases des formes les plus anciennes de coopération internationale ${ }^{1}$.

Cependant, la collaboration dans ce domaine n'a pas toujours été évidente. En effet, l'intérêt pour l'espace marin s'est déclaré très tôt, notamment grâce au commerce qui s'exerçait principalement par voie maritime. C'est ainsi que dès les premiers contacts de l'homme avec cet espace nouveau, s'est posé le problème juridique de la souveraineté sur la mer et des attributs qui en découlent.

Mais ce sont véritablement les progrès techniques du $\mathrm{xx}^{\mathrm{e}}$ siècle, en permettant une connaissance plus approfondie des richesses de la mer et une meilleure exploitation de ses ressources, qui accélérèrent et amplifièrent la nécessité chez les États côtiers d'étendre leur domination économique sur la mer, d'autant plus qu'il s'agissait des dernières possibilités d'appropriation sur le globe terrestre. L'enjeu devenait donc d'une importance capitale et il n'était pas envisageable pour les États de laisser passer une telle source de pouvoir et d'enrichissement. Partant de l'opposition entre deux théories extrêmes issues du droit coutumier, celles du mare clausum et du mare liberum, les États se sont peu à peu tournés vers une troisième voie, construite sous l'égide des notions de partage et d'appropriation étatique.

1. Terry Olson, «Mer et espace: brèves réflexions », Annuaire de droit maritime et océanique (ADM), 1999, p. 227. 
Après une phase de profondes modifications jalonnées d'échecs, le "nouveau droit de la mer" instauré par la convention de Montego Bay de $1982^{2}$ va mettre en place de nouvelles zones maritimes et étendre d'autres espaces auparavant institués par les conventions de Genève de $1958^{3}$. Ainsi, les États côtiers avancent dans un contexte nouveau où les espaces délimités portent sur de plus grandes distances (de 200 à 350 milles marins pour la Zone économique exclusive ZEE - et le plateau continental). Le problème, comme l'explique JeanPaul Pancracio, c'est qu' « en projetant vers le large la souveraineté, la juridiction ou bien des droits souverains des États côtiers, on a suscité des zones de rencontre frontale ou latérale de ces projections, partout où l'espace marin ne permettait pas une pleine extension des zones promises aux États côtiers ${ }^{4}$ ». Dans le même sens, la Cour internationale de justice (CIJ) relèvera dès son premier arrêt en la matière que « s'agissant de la délimitation, le grand problème n'était pas celui des limites entre États mais celui des limites vers le large de l'étendue sur laquelle l'État riverain peut revendiquer des droits d'exploitation exclusifs ${ }^{5}$ ».

C'est pourquoi, consciente du grand nombre de litiges potentiels, la convention de Montego Bay a prévu en sa partie XV le principe de règlement pacifique des différends en matière de délimitation maritime. Si près de 200 délimitations ont jusqu'à aujourd'hui été effectuées par le biais diplomatique de la négociation ${ }^{6}$, d'autres ont tout de même dues être traitées, soit par la voie de l'arbitrage, soit par la voie juridictionnelle. Parmi ce dernier mode de règlement, la CIJ a toujours été le moyen judiciaire privilégié par les États et a tenu un rôle important dans le développement du droit de la mer. En effet, la Cour a effectué un travail d'éclaircissement des dispositions de la convention de Montego Bay, connues pour leur caractère générique. C’est ainsi que dans ce domaine, ses décisions «ont pu être qualifiées de "révélatrices" par certains juristes

2. La convention de Montego Bay a été adoptée en Jamaïque le 10 décembre 1982 et est entrée en vigueur le 16 novembre 1994.

3. Les 4 conventions de Genève portant sur le droit de la mer ont été adoptées le 29 avril 1958. La convention sur la mer territoriale et la zone contiguë est entrée en vigueur le 10 septembre 1964, la convention sur la haute mer le 30 septembre 1962, la convention sur la pêche et la conservation des ressources biologiques de la haute mer le 20 mars 1966 et la convention sur le plateau continental le 10 juin 1964.

4. Jean-Paul Pancracio, Droit de la mer, Paris, Dalloz, 2010, p. 256.

5. CIJ, Plateau continental de la mer du Nord, recueil, 1969, p. 33.

6. Ce qui amène d'ailleurs certains auteurs à se demander si les États n'auraient pas plutôt intérêt à recourir à la conciliation internationale pour les délimitations qu'il reste à effectuer. Voir Richard Meese, «Délimitations maritimes: règlement juridictionnel et conciliation internationale», $A D M, 1998$, p. 161-189. 
dans la mesure où non seulement elles témoignaient de règles de droit existantes, mais également dans le sens où elles pouvaient être regardées comme des sources de droit pour l'avenir ${ }^{7}$ ».

D'autant plus que si les bases du droit de la mer sont anciennes, la délimitation maritime est une activité relativement récente, puisque les premières revendications sur l'étendue des espaces marins sont apparues à partir des années $1950^{8}$ (notamment dans les mers fermées ou semi-fermées bordant de nombreux États, comme la mer du Nord, la Méditerranée, les golfes, etc.), entraînant une augmentation des conflits potentiels et la nécessité pour la CIJ de mettre en place une méthode stable de délimitation.

Mais dans ce domaine, bien que le maitre mot en la matière soit le respect de l'équité, il n'existe pas de procédé unique de délimitation. Cela s'explique par le fait qu'une méthode universelle nécessiterait une définition in abstracto de l'équité, or chaque situation est constituée de particularités géographiques, géologiques, géomorphologiques, politiques et économiques diverses, rendant chaque cas d'espèce difficilement transposable à un autre. D'ailleurs, si la CIJ, dès l'affaire du plateau continental de la mer du Nord de 1969, estime que c'est une « vérité première » que de considérer qu'une délimitation doit être établie de manière équitable, elle précise dans le même temps qu'il faut « rechercher non pas une méthode unique de délimitation mais un but unique ": "le problème est surtout de définir les moyens par lesquels la délimitation peut être fixée de manière à être reconnue comme équitable ${ }^{9}$.» Ainsi, «l'unité de but laisse place à la pluralité des méthodes ${ }^{10}$ ". Néanmoins, s'il n'existe pas de méthode universelle, il est la plupart du temps donné priorité au

7. Paul de Geouffre de La Pradelle, «Notions de territoire et d'espace dans l'aménagement des rapports internationaux contemporains ", Recueil des cours de l'académie de droit international (RCADI), 1977, t. 157-IV, p. 428-433, reprenant Jean-Pierre Queneudec, «The Role of the International Court of Justice and Other Tribunals in the Development of the Law of the Sea ", dans A. H. A. Soons (dir.), Implementation of the Law of the Sea Convention through International Institutions (Proceedings of the 23rd Annual Conference of the Law of the Sea Institute, Honolulu, 1989), 1989, p. 575 .

8. En effet, jusqu'en 1938, la France n'avait effectué que le tracé des lignes de base droites en Méditerranée par la loi du $1^{\mathrm{er}}$ mars 1888 . Puis, par un décret du $1^{\mathrm{er}}$ juin 1938, la France a défini les lignes de base droites au niveau des baies du littoral de la Manche et de l'Atlantique. Ce n'est qu'en 1967 que l'État français a tracé une délimitation maritime avec Monaco, puis avec l'Espagne en 1974.

9. CIJ, Plateau continental de la mer du Nord, op. cit., p. 50.

10. Michel Voelckel, «Aperçu de quelques problèmes techniques concernant la délimitation des frontières maritimes ", Annuaire français de droit international (AFDI), 1979, p. 700. 
procédé de l'équidistance consistant à tracer une ligne à égale distance des côtes des deux États.

Cependant, la justice, aussi équitable et juste soit-elle, a une utilité très limitée si ses décisions, définitives et obligatoires, ne sont pas exécutées ${ }^{11}$. Or il est convenu que :

l'exécution des arrêts internationaux ainsi que l'application des décisions d'organisations internationales ont toujours posé des problèmes graves et difficiles dans les milieux diplomatiques et judiciaires. En effet, les questions touchant à la mise en œuvre des arrêts et des décisions internationaux ont été constamment liées aux questions plus générales concernant l'effectivité $\mathrm{du}$ système international dans son ensemble, ou encore son caractère de véritable ordre juridique ${ }^{12}$.

D'ailleurs, jusqu'en 1967, la France n'avait pas opéré de frontière maritime avec ses États voisins.

Mais quels seraient alors l'intérêt, l'objectif et le rôle réels du droit et de la justice si les décisions des juridictions internationales n'étaient pas exécutées? Dans ce sens, on peut affirmer que l'exécution fait partie intégrante du contentieux, plus encore en droit international, où la mise en œuvre effective des arrêts est son unique moyen d'existence en cas de litige. Cela est d'autant plus vrai dans le cadre des délimitations maritimes qu'il s'agit d'une matière très spécifique à plusieurs niveaux. En effet les affaires y ayant trait relèvent du contentieux des frontières qui, à l'instar du contentieux territorial, induisent des enjeux multiples et essentiels pour les États. Faire accepter à une partie de limiter l'étendue de sa souveraineté et des avantages que la situation antérieure lui conférait n'est donc pas chose aisée, même par voie juridictionnelle.

Malgré l'importance de la question, la phase juridictionnelle étant distincte de la phase post-juridictionnelle, cette dernière n'est quasiment pas abordée dans le cadre des arrêts internationaux, imposant ainsi un triste constat: il existe encore aujourd'hui un manque d'intérêt pour cette facette du droit international. Comme le remarquait déjà Shabtai Rosenne il y a près de soixante ans, « un trait frappant de la littérature traitant du règlement judiciaire des conflits internationaux est son indifférence relative en ce qui concerne les problèmes de l'exécution et

11. Voir Nathalie Ros, «Les effectivités (rôle et place)», dans Le Processus de délimitation maritime. Étude d'un cas fictif (actes du colloque international de Monaco des 27-29 mars 2003), Paris, Pedone, 2004, p. 195-227.

12. Andréa Giardana, «La mise en œuvre au niveau national des arrêts et des décisions internationaux», RCADI, 1979, t. 165-IV, p. 243. 
la mise en vigueur des décisions de la Cour internationale ${ }^{13}$ ", avançant pour justification l'explication selon laquelle après la seconde guerre mondiale, il semblait plus important de s'occuper de la compétence de la Cour que des suites que les États pouvaient ou voulaient donner à la décision rendue à leur encontre. Or analyser ce pan devrait permettre de maîtriser les techniques, les faiblesses et les enjeux de la phase d'exécution afin d'améliorer l'efficacité de la justice internationale.

Paradoxalement, les décisions rendues par la CIJ sont relativement bien mise en œuvre dans l'ensemble ${ }^{14}$ et plus particulièrement dans la matière si sensible des délimitations maritimes, qui n'a pour le moment connu aucun cas de non exécution. C'est probablement une des raisons pour laquelle les moyens d'application des décisions de la CIJ par les parties et les obligations liées à l'exécution ont été très peu étudiées ${ }^{15}$. L'analyse de la rareté des défauts apparaît donc comme un défi. Pourtant, le double constat, qui pourrait a priori paraitre antinomique, entre la sensibilité de la matière et l'exécution néanmoins convenable des décisions de la Cour sur le sujet, mérite d'être étudié plus en détail. Il s'agit en effet de deux points caractéristiques du contentieux de la délimitation maritime, donnant un intérêt particulier à la tentative d'explication du paradoxe de l'exécution satisfaisante des décisions de la CIJ dans cette matière particulièrement délicate.

\section{La délimitation maritime : une matière aux enjeux cruciaux}

La première raison expliquant la volonté des États de s'entendre sur les délimitations, si besoin en ayant recours à la CIJ, est l'ensemble des activités substantielles qui sont effectuées ou susceptibles de l'être dans la zone de conflit par les États côtiers. Un tel litige pourrait conduire

13. Shabtai Rosenne, "L'exécution et la mise en vigueur des décisions de la Cour internationale de justice », Revue générale de droit international public (RGDIP), 1953, p. 532.

14. Voir déclaration du président de la CIJ, Hisashi Owada, « $6^{\mathrm{e}}$ Commission : le Président de la CIJ se dit préoccupé des obstacles politico-juridiques qui empêchent les États d'exécuter les arrêts de la Cour", 29 octobre 2006, AG/J/3403, (document non officiel), disponible sur http://www.un.org/News/fr-press/docs/2010/AGJ3403.doc. htm [consulté le 25 août 2012].

15. Voir Affef Ben Mansour, La mise en ceuvre des arrêts et des sentences des juridictions internationales, thèse de l'université Paris-Ouest - Nanterre-La Défense, Alain Pellet (dir.), 2009, $566 \mathrm{p}$. 
à une baisse non négligeable des intérêts économiques ainsi qu'à des divergences politiques pouvant amener à un véritable conflit armé ${ }^{16}$.

En effet, l'emplacement de la ligne frontière, en tant que «limite en mer de la souveraineté d'un État côtier ${ }^{17}$ ", déterminera, d'une part, l'étendue de la juridiction de l'État ${ }^{18}$ et, d'autre part, l'étendue des droits de l'État sur les ressources biologiques et minérales. En ce sens, en plus de constituer un enjeu économique majeur, la délimitation maritime revêt aussi un caractère politique puisqu'elle n'est pas naturelle; elle est le fruit de la volonté des États ${ }^{19}$. Il existe également des considérations stratégiques, concernant la sécurité, la protection des intérêts de la navigation, etc., sans oublier de prendre en considération les facteurs historiques. Bien que ces derniers ne constituent pas des "enjeux » à proprement parler, ils sont souvent rattachés à d'autres facteurs, économiques ou politiques. C'est le cas par exemple des baies historiques qui sont utilisées à des fins de pêche par l'État côtier. Or ces habitudes constituent autant de prétentions dans les revendications étatiques portées devant la CIJ. C'est d'ailleurs une des raisons pour laquelle la pratique antérieure des États est souvent prise en compte par la Cour en tant que circonstances spéciales. Dans une moindre mesure, les considérations environnementales, bien que leurs effets soient souvent considérés comme mineurs, peuvent aussi faire l'objet de revendications.

Mais enfin et surtout, les délimitations, quelles soient terrestres ou maritimes, constituent un aspect émotionnel, psychologique, très fort pour les États. Ce constat découle de l'ensemble des enjeux jusqu'ici évoqués. C'est pourquoi la question de l'exécution des arrêts de la CIJ en la matière, bien qu'elle soit relativement positive, est néanmoins sensible et loin d'être une évidence.

Donc, bien que certains estiment encore que les délimitations maritimes sont moins délicates que l'établissement des frontières terrestres, les enjeux sont indéniables et constituent des avantages essentiels aux yeux des États. De plus, les difficultés techniques de mise en œuvre semblent plus importantes pour le cas des frontières maritimes que pour

16. Voir Bernard H. Oxman, "Political, Strategic, and Historical considerations ", dans J. I. Charney et L. M. Alexander (dir.), International Maritime Boundaries, Dordrecht-Boston-Londres, Martinus Nijhoff Publisher, 1999, vol. 1, p. 3-40.

17. Jean Salmon, "Délimitation maritime», dans Dictionnaire de droit international public, Bruxelles, Bruylant, 2001, p. 523.

18. Voir Laurent Lucchini et Michel Voelckel, Droit de la mer, Paris, Pedone, 1996, t. II (Délimitation, navigation et pêche), vol. 1 (Délimitation), p. 24, \$560.

19. Prosper Weil, Perspectives du droit de la délimitation maritime, Paris, Pedone, 1988, p. 35 . 
les frontières terrestres ${ }^{20}$. Malgré tout, les arrêts de la CIJ en matière de délimitation maritime sont exécutés de manière satisfaisante.

\section{Le constat de l'exécution satisfaisante des arrêts de la CIJ en matière de délimitation maritime}

C'est probablement une des raisons expliquant pourquoi la CIJ, comme la CPJI, ont continuellement refusé de se pencher sur la possibilité de l'inexécution de leurs décisions, partant du postulat que le consentement des États à saisir la Cour constitue en soit une garantie d'exécution ${ }^{21}$. Il est vrai que dans l'observation de la pratique, seule une poignée d'arrêts, en plus de soixante ans d'existence de la Cour, ont connu de réelles difficultés d'application.

Mais s'ajoute un élément essentiel à l'exécution effective des décisions de la CIJ : la volonté des États parties au différend, plus ou moins enclins à mettre en ouvre une décision qui les condamne, les affecte ou ne correspond pas exactement à leurs prétentions initiales. Cet élément introduit une variable rendant en définitive l'exécution beaucoup moins simple et linéaire qu'on pourrait le penser et, si les cas de réelle inexécution sont extrêmement rares, la Cour a tout de même d'ores et déjà été confrontée à la mauvaise volonté des États avant de voir son arrêt mis en œuvre.

En effet, une «bonne exécution » ne désigne pas uniquement le résultat de l'application effective de l'arrêt issu d'une décision internationale, elle renvoie aussi et surtout à un processus de mise en œuvre. Quand la $\mathrm{CIJ}$ est saisie, son but est de résoudre le litige entre les États dans l'objectif de maintenir la paix internationale. Leur bonne volonté et leur bonne foi va alors prendre une place très importante car c'est véritablement le comportement des États dans la phase post-juridictionnelle qui va permettre d'anéantir les tensions préexistantes. Dans cette optique, la "bonne exécution" des décisions internationales correspond à la manière dont les États vont exécuter l'arrêt. N'oublions pas que lors de l'affaire de la frontière terrestre et maritime entre le Cameroun et le Nigéria $^{22}$, l'attitude hostile du Nigeria donna lieu à un nouveau conflit. Une bonne exécution sera donc une exécution de bonne foi, donnant lieu

20. Michel Voelkel, «Aperçu de quelques problèmes techniques concernant la délimitation maritime», art. cité, p. 695.

21. Aïda Azar, L'Exécution des décisions de la Cour internationale de justice, Bruxelles, Bruylant, 2003, p. 50.

22. CIJ, Frontière terrestre et maritime entre le Cameroun et le Nigeria (Cameroun c. Nigeria; Guinée équatoriale, intervenant), recueil, 2002. 
à une mise en œuvre efficace et effective, permettant de mettre réellement fin au litige.

La difficulté, dans le cadre de l'exécution des décisions internationales, c'est que la mise en œuvre du jugement est véritablement entre les mains des États parties au différend, puisque contrairement à l'ordre juridique national, les juridictions internationales ne disposent pas du recours à la force publique pour faire exécuter leur décision en cas d'obstacle à l'exécution ${ }^{23}$. En réalité, «les États sont conscients de leur obligation de se conformer aux décisions de la Cour » : « Les plus grandes difficultés se posent bien souvent lorsqu'il s'agit pour les États d'honorer effectivement leur obligation découlant de l'arrêt de la $\operatorname{Cour}^{24}$.»

Un premier élément de réponse au paradoxe de l'exécution quasisystématique des arrêts de la CIJ se trouve dans le fait que la volonté des États parties au différend est respectée au point que l'on peut affirmer qu'elle « encadre » véritablement l'ensemble de la procédure juridictionnelle. Il est vrai que la CIJ a été créée dans la conscience que «bien qu'acquis aux idéaux de la paix, l'environnement de l'époque n'en était pas moins réfractaire à tout supranationalisme »: «La souveraineté de l'État demeurait plus que jamais le terreau dans lequel pouvait seulement germer une institution intergouvernementale de coordination, c'està-dire sans prétention supranationale ${ }^{25}$.» Et, «bien qu'ayant évolué, la société internationale n'est, aujourd'hui pas plus qu'hier, prête pour un système de juridiction obligatoire ${ }^{26} »$.

C'est pourquoi, dans un premier temps, l'article $36, \$ 2$ du statut de la Cour a subordonné son action au consentement préalable de l'État. Elle ne peut donc pas s'autosaisir; cela représenterait une ingérence dans les affaires des parties qui amènerait très probablement à un rejet de l'intervention de la CIJ ainsi qu'à un refus d'exécuter la décision internationale. Ainsi, l'indispensable consentement des États dans la

23. Hishashi Owada le formule très justement: «En droit interne, l'État a l'autorité et les moyens d'exécuter un jugement. En revanche, l'exécution des arrêts de la Cour, réglée par l'article 94 de la Charte des Nations unies, dépend de l'initiative de l'une des parties à l'affaire, qui peut décider de saisir le Conseil de sécurité si l'autre partie ne s'acquitte pas d'une obligation résultant de l'arrêt. Le Conseil de sécurité, s'il le juge nécessaire, peut alors faire des recommandations ou décider des mesures à prendre en vertu des chapitres vi et viI de la Charte. L'exécution relève donc de la compétence du Conseil de sécurité » (déclaration citée supra).

24. Ibid.

25. Communication de Mohammed Bedjaoui lors de la $66^{\mathrm{e}}$ session de droit international public de l'Académie de droit international de La Haye, "Le cinquantième anniversaire de la Cour internationale de Justice», RCADI, 1996, p. 21, 23.

26. Ibid., p. 21, $\$ 24$. 
phase pré-juridictionnelle montre déjà une forme d'encadrement de la procédure. D'autant plus qu'elle ne peut le présumer.

Mais une fois la compétence de la Cour acceptée, la volonté étatique va continuer son œuvre puisque dans le compromis, les États parties vont également pouvoir déterminer la mission des juges; ayant pour conséquence de régir le cours du procès et, indirectement, de maîtriser le fond de la procédure. Ces instruments seront plus ou moins détaillés, offrant aux juges une marge de manœuvre proportionnelle à la rigueur des formulations ${ }^{27}$. Et la Cour ne pourra pas outrepasser ses pouvoirs. Autrement dit, les parties indiquent à la CIJ le droit applicable. Bien que cette tendance n'ait pas toujours été admise dans les instances internationales, la Cour l'a acceptée afin de rendre une décision mieux acceptée des États en cause puisqu'elle sera fondée sur l'accord entre les parties.

Enfin, c'est de manière toujours plus consensuelle et soucieuse de l'acceptation de ses décisions par les États parties au différend, particulièrement en matière de délimitation maritime, que la Cour va permettre à ces derniers de renégocier les modalités de mise en œuvre ${ }^{28}$. Cette nouvelle période de confrontation débouchera soit sur un accord, soit sur une autre action contentieuse. Que cet élément soit le fruit d'une stratégie active de la Cour ou bien un signe d'affaiblissement de la force obligatoire de ses arrêts, cela montre avant tout la force du volontarisme étatique donnant lieu à une maîtrise de la procédure et une relative liberté dans la phase post-juridictionnelle permettant d'expliquer le paradoxe de l'acceptation et de l'exécution des décisions de la CIJ dans une matière aussi sensible que les délimitations maritimes.

27. En pratique, dans ces instruments, les États parties au différend peuvent formuler trois types de requêtes auprès des juges de la $\mathrm{CIJ}$ : déterminer précisément le tracé de la ligne de démarcation de la délimitation maritime, déterminer uniquement les principes de la délimitation à effectuer, ou encore réaliser une demande quelque peu hybride. Ce fut par exemple le cas de l'article 2 du compromis du 10 juin 1977 entre la République tunisienne et la Jamahiriya arabe libyenne qui demandait à la CIJ de préciser «les principes et règles du droit international qui peuvent être appliqués pour la délimitation ", mais en demandant également de "clarifier avec précision la manière pratique par laquelle lesdits principes et règles s'appliquent dans cette situation précise, de manière à mettre les experts des deux pays en mesure de délimiter lesdites zones sans difficultés aucunes ».

28. En matière de délimitation maritime, l'exemple le plus significatif est celui de l'accord entre le Danemark et la Norvège en date du 18 décembre 1995 faisant suite à l'arrêt rendu par la CIJ dans l'affaire de la délimitation maritime dans la région située entre le Groenland et Jan Mayen du 14 juin 1993 (voir CIJ, Délimitation maritime dans la région située entre le Groenland et Jan Mayen, recueil, 1993, p. 38). Ce dernier modifiera les coordonnées de la ligne de délimitation proposée par la CIJ. 
En définitive, il est tout aussi intéressant d'étudier un bilan plutôt positif; nous amenant à analyser les raisons d'un tel constat, ainsi que l'opportunité d'améliorer encore le système. Ces interrogations sont d'autant plus pertinentes concernant l'exécution des décisions du contentieux de la délimitation maritime qu'il s'agit d'une matière touchant à la souveraineté des États et à de nombreux enjeux.

De plus, si frontières terrestres et maritimes peuvent avoir des traits communs, leurs différences, notamment dans l'exécution, sont éclatantes. Si pour la première, il est aisé de rendre la frontière visible (et donc effective), dans le domaine maritime en revanche, on ne peut pas réellement parler de "démarcation", car les délimitations ne permettent aucun repère physique visible. Dans ce cas, la question se pose de savoir s'il est possible de rendre effective une décision en matière de délimitation maritime. Une décision délimitant une zone maritime peut-elle être considérée comme réelle et effective alors que son exécution porte sur de l'immatériel?

Sachant que pour réaliser les délimitations qui lui sont demandées, la CIJ utilise des moyens spécifiques de règlement des différends, tel le principe de l'équité. Outre le fait que ces règles et techniques permettent en partie de répondre aux questions évoquées ci-dessus et de les intégrer dans le cadre de l'étude ici réalisée, elles appellent également à se demander si la flexibilité du raisonnement de la Cour suffit pour faire accepter et respecter ses arrêts ou si, au contraire, elle ne nuirait pas en réalité à la force de ses décisions.

En effet, le président de la CIJ a constaté l'affaiblissement de la force obligatoire due à la volonté des États dans l'exécution. Ce constat est également lié à un des problèmes majeurs de la justice internationale; le fait qu'il n'existe pas dans l'ordre juridique international de force publique obligeant à l'exécution des arrêts. Par ailleurs un problème structurel fait que la Cour ne peut assurer elle-même l'exécution de ses arrêts. En outre, la délimitation maritime touchant de près ou de loin - selon qu'il s'agisse de la mer territoriale ou de la zone économique exclusive (ZEE) et du plateau continental - à la souveraineté des États, la CIJ peut-elle ainsi toucher à la raison d'être de l'État sans que cela ne porte préjudice à la mise en œuvre de ses arrêts? Car il s'agit bien dans ce domaine d'une immixtion de la Cour dans l'étendue de l'imperium de l'État.

Pour autant, les décisions en matière de délimitation ne posent généralement pas de problème en termes d'exécution. Dans la plupart des cas, les États acceptent sans trop de difficultés une décision touchant à l'étendue de leur souveraineté. La CIJ arrive donc à imposer des 
décisions donnant lieu à une exécution immatérielle, limitant l'étendue des pouvoirs souverains d'au moins une des parties au différend et de leur côté, les États exécutent convenablement les décisions de la Cour. Quels éléments peuvent expliquer ce paradoxe? Les États sont-ils aussi libres dans l'exécution de ce genre de décision que leur maitrise de la procédure juridictionnelle semble le laisser croire? Il existe bien une obligation d'exécution des arrêts de la Cour à la charge des États parties au différend. Comment cette dernière est-elle appliquée en pratique? Cette « contrainte» suffit-elle à assurer une mise en œuvre satisfaisante de la décision?

Autrement dit, il convient de se demander pourquoi les arrêts de la CIJ, dans une matière aussi sensible que les délimitations maritimes, font dans l'ensemble l'objet d'une exécution exemplaire. Dans quelle mesure des décisions aussi délicates que celles rendues en matière de délimitation maritime sont-elles acceptées et exécutées par les États parties au différend?

Il apparaît en effet que les États parties au différend ne sont pas tout à fait libres dans la phase d'exécution de la décision de la CIJ. Si les parties ont une maitrise incontestable de la procédure juridictionnelle, il existe cependant certaines garanties permettant d'assurer que ces dernières exécuteront l'arrêt de la Cour, d'autant plus que la pratique actuelle montre une tendance forte à un encadrement de plus en plus prononcé de la volonté des États, limitant largement leur marge de manœuvre dans la phase post-juridictionnelle.

\section{Une exécution assurée par des garanties multiformes}

Contrairement à la liberté qui semble être laissée aux États parties à ce type de différend du fait de leur maîtrise de la procédure, «le droit international n'est pas tout entier un droit volontaire, et la volonté de l'État n'est pas la source unique des obligations qui sont à sa charge ${ }^{29}$ ". D'ailleurs, afin de garantir une exécution satisfaisante des arrêts de la CIJ dans une matière aussi sensible que les délimitations maritimes, il serait considéré comme inopportun d'accorder toute confiance à la bonne volonté des États parties au différend; leur susceptibilité dans les domaines touchant à la souveraineté étant largement exacerbée. C'est pourquoi des garanties préventives ont été mises en place; tantôt en amont lorsqu'il s'agit pour la Cour de faire accepter sa décision au travers

29. Maurice Kamto, «La volonté de l'État en droit international», RCADI, t. CCCX, 2004, p. 427. 
d'une démarche inédite; tantôt en aval lorsqu'il est question d'encadrer la liberté des parties dans la phase d'exécution.

\subsection{Le consensualisme de la CIJ ou la recherche de l'acceptation de la décision}

En tant qu' « opération juridico-politique ${ }^{30}$ » déterminant « une composante capitale de l'État ${ }^{31} »-$ l'étendue de sa souveraineté en mer -, la délimitation d'une frontière maritime est une opération délicate pour les juges de la CIJ. Il est en effet impossible pour ces derniers de faire abstraction des intérêts en jeux, d'autant plus que les affaires portées à sa connaissance sont souvent les plus sensibles puisqu'elles n'ont pas pu être résolues par voie diplomatique entre les États. Afin que les parties au litige adhèrent à sa décision, la Cour va donc devoir faire preuve de consensualisme. Cette recherche de compromis s'inscrit dans la lignée de l'article $33 \$ 1$ de la Charte des Nations unies qui prône en premier lieu le recours à la négociation en cas de différend. Or dans le cadre du contentieux de la délimitation maritime, cette négociation va se faire entre les États opposés et la Cour, permettant ainsi d'instaurer un climat propice à l'acceptation de l'arrêt de la CIJ et assurant ensuite son exécution. Ce particularisme est tellement spécifique au contentieux territorial qu'il est aujourd'hui considéré comme «le plus saillant des traits essentiels caractérisant sa fonction ${ }^{32}$ ».

Dans l'optique de mettre en ouvre cette philosophie consensuelle, la Cour va chercher à parvenir à un résultat juste et donc acceptable et a, pour ce faire, mis en place une méthode spécifique à ce contentieux. En effet, le juge, devant uniquement trancher sur la base du droit, se trouve en matière de délimitation maritime confronté aux lacunes des dispositions des conventions de Genève et de Montego Bay sur le plateau continental ou la ZEE. Une précision fut néanmoins apportée par la convention de Montego Bay - mais il s'agit d'une précision essentielle dans la mesure où elle inscrit le contentieux de la délimitation maritime

30. CIJ, Délimitation de la frontière maritime dans la région du golfe du Maine, recueil, 1984, p. $246, \$ 55$.

31. Laurent Lucchini, «La délimitation des frontières maritimes dans la jurisprudence internationale: vue d'ensemble», dans R. Lagoni et D. Vignes, Maritime Délimitation, Martinus Nijhoff Publishers, «Publications on Ocean Development », 2006, p. 3 .

32. Mohammed Bedjaoui, art. cité, p. 21, $\$ 23$. 
dans une "politique judiciaire de transaction ${ }^{33}$ » - selon laquelle la délimitation du plateau continental ou de la ZEE entre États dont les côtes sont adjacentes ou se font face doit être effectuée dans le but d'obtenir un résultat équitable (articles 74 et 83 de la convention de Montego Bay). De cette règle va découler le principe coutumier d'équité consistant en "l'obligation de négocier, et de faire de telle sorte que cela ait quelque chance d'aboutir à un accord ${ }^{34}$ ». La Cour va donc devoir aller au-delà de la simple juris dictio et se placer plutôt dans un rôle d'arbitre. Par ailleurs, si «l'équité n'implique pas nécessairement l'égalitée ${ }^{35}$ ", elle peut avoir un rôle correctif, dans le sens où elle est susceptible de diminuer les rigidités et effets inéquitables de la $\mathrm{loi}^{36}$. Dans cette optique, l'équité, en tant que moyen de parvenir à un résultat équitable, traduit la philosophie consensuelle de la Cour ${ }^{37}$.

Afin de juger en équité et d'atteindre son objectif, la Cour a, au fur et à mesure des différentes affaires en la matière portées à sa connaissance, clarifié son raisonnement, notamment en privilégiant l'équidistance qui, selon elle, permet de respecter l'égalité entre les États ${ }^{38}$. La technique de l'équidistance, seule méthode mentionnée dans les conventions de Genève, consiste en «la construction géométrique d'une ligne à partir de points - à définir - dont cette ligne doit être équidistante ${ }^{39}$ ". Il est d'ailleurs observable que, dans la pratique des délimitations maritimes, le juge ne suivra la plupart du temps aucune des lignes respectivement défendues par les parties, car la solution risquerait de léser trop fortement l'autre partie, amenant à une situation de gagnantperdant qui limiterait l'acceptation de la décision. Au contraire, à la manière d'un jugement de Salomon, la Cour cherchera plutôt un « juste milieu $^{40} »$ entre les différentes prétentions afin de rendre acceptable une

33. Géraldine Giraudeau, Le juge international et le règlement transactionnel des différends territoriaux, thèse, université Paris I - Panthéon-Sorbonne, Pierre-Michel Eisemann et Carlos R. Fernandez Liesa (dir.), 2010, p. 149.

34. François Monconduit, "Affaire du Plateau continental de la mer du Nord, RFA c/ Danemark, RFA c/ Pays-Bas - arrêt du 20 février 1969 ", AFDI, 1969, p. 238.

35. Ibid., p. 240.

36. Voir L. M. D. Nelson, "The role of equity in the delimitation of maritime boundaries", American Journal of International Law (AJIL), vol. 84, $\mathrm{n}^{\circ}$ 4, p. 839-841.

37. Voir Wolgang Friedmann, «The North Sea Continental Shel Cases - a Critique », AJIL, 1970, p. 236.

38. Voir Laurent Lucchini, «Le contentieux de la délimitation maritime et l'apport de la jurisprudence», dans Institut du droit économique de la mer, Le Plateau continental dans ses rapports avec la zone économique exclusive, Paris, Pedone, 2005, p. 25.

39. Michel Voelckel, «Aperçu de quelques problèmes techniques... », art. cité, p. 702.

40. Géraldine Giraudeau, op. cit., p. 152. 
décision qui ne satisfera pleinement aucune des deux parties au différend (voir la carte ci-dessous). Cette méthode conduit souvent à des résultats plus équitables dans les cas simples et aboutit à des tracés plus clairs que ceux résultant de certains autres procédés ${ }^{41}$.

D'autre part, toujours dans un souci de clarification, la Cour va adopter un "processus stable de délimitation ${ }^{42}$ ", selon lequel les juges devront d'abord tracer une ligne provisoire d'équidistance, puis, si cette ligne apparaît avoir un effet équitable, alors elle devient définitive; dans le cas contraire, les juges procéderont à un ajustement en prenant en considération les circonstances qu'ils estiment pertinentes ${ }^{43}$. Cet éclaircissement a été primordial pour les États, car il entraîne une grande sécurité juridique. En effet, en connaissant par avance la démarche adoptée par la Cour, la solution sera plus ou moins prévisible, et donc, à partir du moment où des États décideront de porter une affaire devant la Cour, c'est qu'ils auront la volonté d'exécuter l'arrêt qui en découlera ${ }^{44}$.

Mais si la ligne d'équidistance est le moyen privilégié, ce n'est pas le seul utilisé dans la délimitation; l'équité n'exigeant pas obligatoirement le recours à cette méthode pour effectuer une délimitation maritime, car elle peut dans certains cas aboutir à une iniquité. La Cour estime donc qu'une méthode de délimitation unique ne peut pas être satisfaisante dans toutes les circonstances et qu'il faut alors s'adapter au cas par cas pour que la solution soit équitable et donc acceptée par les parties. À ce propos, dès l'affaire du plateau continental de la mer du Nord de 1969, la CIJ affirme que «l'emploi de cette méthode peut, dans certains cas aboutir à des résultats de prime abord extraordinaires, anormaux ou déraisonnables (dans ce sens, la République fédérale a présenté une carte de l'océan Atlantique découpé selon le principe d'équidistance, ce qui notamment aboutit à donner à la France une part minuscule, tandis que celle du Portugal est étrangement étendue ${ }^{45}$ ) ». La CIJ continue d'ailleurs de tenir le même raisonnement dans l'affaire de la délimitation maritime en mer Noire (Roumanie/Ukraine) du 3 février 2009 - son dernier arrêt - où elle indique que « lorsqu'il s'agit de procéder à une délimitation

41. Michel Voelckel, art. cité, p. 703.

42. Ibid., p. 704 .

43. Pour plus d'informations sur les principes généraux dégagés par la CIJ en matière de délimitation maritime, voir Guiseppe Cataldi, «La ligne unique de délimitation? Application en Méditerranée ", ADM, 1999, p. 229.

44. Sur la prévisibilité des décisions de la CIJ, voir Géraldine Giraudeau, op. cit.

45. CIJ, Plateau continental de la mer du Nord, op. cit..p. 24, \$24. 


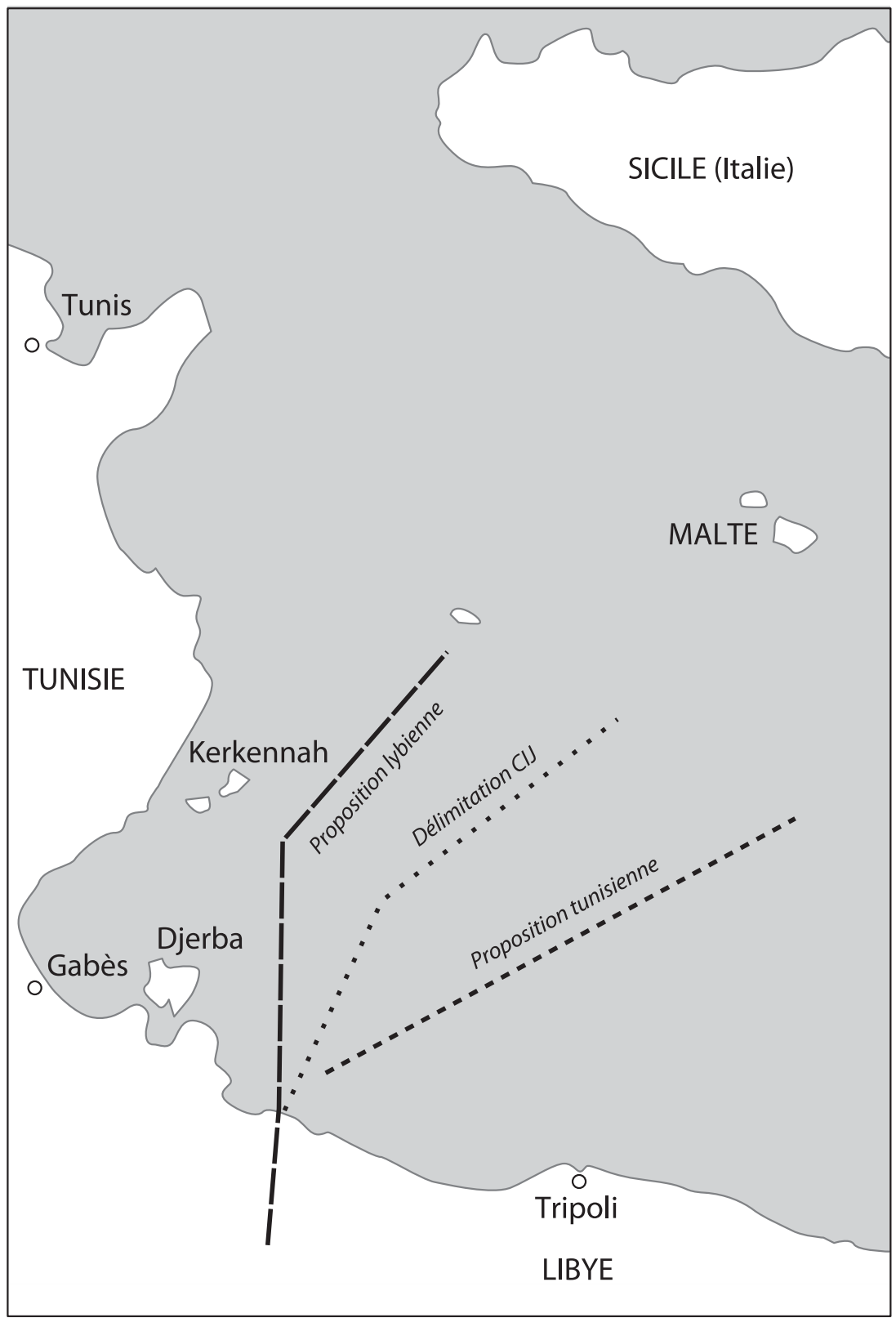

Arrêt CIJ du 24 février 1982, Plateau continental (Tunisie c. Jamahiriya arabe libyenne). Source: Jean-Paul Pancracio, Droit de la mer, op. cit., p. 276. 
entre côtes adjacentes, une ligne d'équidistance est tracée, à moins que des raisons impérieuses propres au cas d'espèce ne le permettent pas ${ }^{46}$ ».

Ce discours explique également la raison pour laquelle la Cour utilise parfois simultanément plusieurs méthodes de délimitation. Dans l'arrêt sur le plateau continental opposant la Tunisie à la Libye, la CIJ, après avoir mis de côté la méthode de l'équidistance ainsi que les méthodes proposées par les parties, va considérer "qu'il n'existe pas en droit international de méthode de délimitation obligatoire et que l'on peut appliquer plusieurs méthodes dans une même délimitation ${ }^{47}$ ». Elle va plutôt utiliser le critère de la proportionnalité ainsi que la technique du "demi-effet », permettant de limiter les déformations pouvant découler de la méthode de l'équidistance sur des longues distances et qui, en l'espèce, donnait un poids excessif à une île. Ce qui signifie bien que pour la Cour, la méthode de l'équidistance n'est pas le seul et unique procédé permettant de parvenir à une solution équitable. Le consensualisme de la Cour, à travers sa recherche du résultat juste et équitable, va alors porter sur différents points : le comportement des parties, le tracé de la ligne de délimitation ou encore la situation existante antérieurement à la saisine de la Cour ${ }^{48}$. Or l'intérêt porté à ces éléments et la manière de les prendre en compte constituent de la part de la CIJ une véritable stratégie pour s'assurer que ses décisions seront bien exécutées.

Dans une matière aussi sensible que les délimitations maritimes, les impératifs liés au consentement des États parties au différend et à la décision de la CIJ vont entraîner cette dernière à adapter sa manière de raisonner aux spécificités de ce contentieux et d'aller au-delà de la simple juris dictio, son objectif n'étant plus uniquement de trancher le litige sur la base du droit mais surtout de faire accepter sa décision. Il ne s'agit donc pas pour la Cour d'être dans un rôle répressif mais plutôt d'agir en termes de compromis. Ainsi, le consensualisme affiché par la $\mathrm{CIJ}$ et qui pouvait de prime abord sembler être une faiblesse - puisque d'un certain point de vue, on peut y voir une certaine soumission de la Cour face aux prétentions des parties au différend - apparait donc en réalité comme une stratégie garantissant doublement l'acceptation

46. CIJ, Délimitation maritime en mer Noire (Roumanie c. Ukraine), recueil, 2009, p. 44, $\$ 116$.

47. CIJ, Plateau continental (Tunisie c. Jamahiriya arabe libyenne), recueil, 1982, p. 18 et p. $79, \$ 111$.

48. Voir respectivement, pour exemple, CIJ, Plateau continental (Jamahiriya arabe libyenne c. Malte), recueil, 1985, p. 19, \$24; CIJ, Délimitation maritime et questions territoriales entre Qatar et Bahreïn, recueil, 2001, p. $57 \$ 173$; CIJ, Délimitation maritime en mer Noire, op. cit., p. 63, $\$ 177$. 
de sa décision puis son exécution, d'une part en assurant une certaine prévisibilité du résultat, et d'autre part en n'aboutissant pas à une situation de gagnant-perdant.

Cependant, cette assurance préalable n'est pas toujours suffisante face à la susceptibilité des États qui, en matière d'étendue de leur souveraineté, peut se trouver exacerbée. C'est pourquoi, afin d'assurer au mieux une exécution satisfaisante, il existe également des garanties post-juridictionnelles limitant la possibilité pour les États de laisser place à une certaine mauvaise volonté d'exécuter un arrêt qui, s'il ne laisse pas un État totalement perdant, n'en satisfait pleinement aucun non plus.

\subsection{L'obligation d'exécuter de bonne foi ou la limite à la liberté des États dans la phase post-juridictionnelle}

Selon l'avis consultatif rendu par la CIJ relatif aux effets des jugements du tribunal administratif des Nations unies, «l'obligation de se conformer aux décisions tant arbitrales que juridictionnelles est considérée comme une règle du droit international général ${ }^{49}$ ». S' il est vrai que les États disposent d'une large marge de manœuvre dans l'exécution des décisions de la CIJ, il est néanmoins nécessaire que cette liberté soit encadrée afin de garantir une exécution efficace. Bien que les parties au différend aient fait le choix de recourir à la Cour - ce qui pose une présomption de bonne volonté de leur part dans la mise en œuvre de l'arrêt et que de son coté la Cour œuvre pour effectuer un compromis entre les différentes prétentions, il est cependant toujours difficile d'accepter une sentence ne correspondant pas exactement à leurs attentes respectives.

À présent, le procédé qui va permettre d'assurer l'exécution va consister en ce que les États n'auront pas le choix d'exécuter ou non la décision; l'obligation d'exécution des arrêts de la Cour étant une obligation juridique, elle s'impose aux parties au litige à partir du moment où ils acceptent la compétence de la juridiction. Malgré la possibilité pour les États parties au différend d'exercer des recours en interprétation et en révision (respectivement, en vertu des articles 60 et 61 du statut de la CIJ), les arrêts de la Cour sont dotés de l'autorité de la chose jugée et seront, à ce titre, considérés comme définitifs (article 60 du statut de la CIJ) et obligatoires pour les parties au différend (article 59 du statut de la CIJ), obligeant de ce fait les États à s'y conformer ${ }^{50}$.

49. Aïda Azar, op. cit., p. $27, \$ 33$.

50. Gilbert Guillaume, «De l'exécution des décisions de la Cour internationale de Justice ", Revue suisse de droit international et européen, 1997, p. 174. 
Cette obligation d'exécution des arrêts de la CIJ est également posée à l'article $94 \$ 1$ de la Charte des Nations unies, selon lequel « chaque membre des Nations unies s'engage à se conformer à la décision de la Cour internationale de justice dans tout litige auquel il est partie». Il s'agit d'une véritable obligation juridique puisqu'en plus d'être consignée dans un instrument conventionnel, elle va également créer des liens de droit entre les parties au différend et la Cour ${ }^{51}$.

Ce lien va s'exprimer à travers deux types de configurations qui donneront néanmoins lieu à la même exigence: mettre en œuvre la décision de la CIJ. Dans un rapport horizontal, l'obligation juridique va découler du lien créé entre les États parties au différend par le prononcé de l'arrêt, car du fait de l'autorité de la chose jugée, lesdits États vont être obligés de mettre en œuvre la décision. Dans un rapport vertical, ce lien va s'établir dans la soumission de l'État à la CIJ du fait de la reconnaissance de compétence effectué avant la phase juridictionnelle ${ }^{52}$. De ce rapport découlera, pour les États parties au différend, une obligation juridique inconditionnelle et immédiate de mettre en œuvre l'arrêt dès son prononcé. Les parties n'auront donc pas à donner leur consentement à l'exécution de la décision; ce dernier ayant été reçu dès l'acceptation de compétence de la Cour. Sinon, cela reviendrait à considérer que l'arrêt n'est pas obligatoire à la base, ce que fit la Libye dans l'affaire du plateau continental (Tunisie c. Libye), où elle ne prenait pour référence que le compromis pour justifier de la force obligatoire de l'arrêt, alors que cette exigence est posée dans la Charte des Nations unies ${ }^{53}$. Cette obligation, issue de la soumission susvisée, sera à l'origine de l'obligation d'exécution de la décision de la Cour.

Il faut cependant préciser que l'obligation de mettre en œuvre l'arrêt de la Cour est différente de l'obligation découlant de l'objet du différend. L'obligation juridique d'exécuter la décision internationale est autonome de l'obligation générale. Quand la Cour rend une décision sur une délimitation maritime, l'obligation de mettre en œuvre le jugement va perdurer jusqu'à ce qu'elle soit effective, c'est-à-dire jusqu'à ce que les États parties au différend aient effectivement mis en œuvre la délimitation maritime dans leur ordre juridique interne. C'est seulement lorsque l'arrêt sera exécuté qu'interviendra l'obligation générale de respecter la limite effectuée, l'arrêt tirant sa force obligatoire de cette obligation d'exécution.

51. Affef Ben Mansour, op. cit., p. 38, \$38.

52. Aïda Azar, op. cit., p. 56, $\$ 60$.

53. Pour cet exemple, ibid., p. $76, \$ 89$. 
Aussi, l'obligation de mise en œuvre étant contenue dans un instrument conventionnel, les États parties à la Charte et recourant à la CIJ doivent, en vertu de la règle pacta sunt servanda, obéir à certaines obligations contenues dans les conventions, comme celle d'exécuter les arrêts de la Cour. Or la Charte, comme la Cour, présument que l'État est respectueux de ses obligations conventionnelles ${ }^{54}$. Bien que "le problème de l'exécution et de la mise en vigueur des décisions judiciaires internationales ne [puisse] guère être abordé sur la base des présomptions de droit affirmant l'observance, par les États, de leurs engagements conventionnels, parce que le problème ne se pose que lorsque la conduite des États, en fait, dévie et ne suit pas la ligne de conduite tracée dans leurs obligations juridiques ${ }^{55}$ », ce cas de figure reste tout de même rare. En définitive, les États ayant recours à la CIJ ont déjà accepté que l'arrêt leur soit obligatoire. À ce propos, la question de l'exécution des arrêts de la CIJ en matière de délimitation maritime, si elle soulève divers problèmes, reste plutôt satisfaisante, ce qui n'est pas le cas de certaines autres affaires. À titre d'exemple dans un autre domaine, la phase post-juridictionnelle de la célèbre affaire des activités militaires et paramilitaires au Nicaragua ${ }^{56}$ a totalement été omise puisque les réparations dues par les États-Unis n’ont jamais été versées.

D'autre part, et surtout, l'obligation d'exécuter les arrêts de la Cour est doublée d'un critère qualitatif de bonne foi dans la mise en œuvre du jugement. Autrement dit, si les États exécutent les arrêts, c'est parce qu'il y a une obligation de mise en œuvre, et s'ils l'exécutent de manière satisfaisante - en ce sens que le conflit est définitivement réglé par le fait que les États parties au différend aient réussi à se mettre d'accord sur les suites à donner à l'arrêt - c'est grâce à l'obligation d'appliquer l'arrêt de bonne foi.

Cette exigence est prévue à l'article $2 \$ 2$ de la Charte, faisant implicitement référence aux obligations découlant des arrêts de la Cour, selon lequel «les membres de l'organisation, afin d'assurer à tous la jouissance des droits et avantages résultant de leur qualité de membre, doivent remplir de bonne foi les obligations qu'ils ont assumées aux

54. Voir Shabtai Rosenne, art. cité, p. 535.

55. Ibid., p. 538.

56. CIJ, Activités militaires et paramilitaires au Nicaragua et contre celui-ci (Nicaragua c. États-Unis d'Amérique), recueil, 1986. 
termes de la présente Charte ${ }^{57}$ ». Bien que cette obligation soit marquée par des valeurs morales, sa présence récurrente dans les textes en fait une véritable obligation juridique ${ }^{58}$. Cependant, il ne s'agit pas d'une source d'obligation autonome, celle-ci étant forcément rattachée à une autre obligation, comme c'est le cas de l'obligation de négocier de bonne foi, ou encore, dans le cadre de notre étude, de l'obligation d'exécuter les arrêts de la CIJ de bonne foi. En outre, il s'agit du corollaire du consentement préalable de l'État à saisir la Cour, puisqu'en agissant ainsi, les États sont présumés accepter le jugement par avance et donc avoir consenti à l'exécuter de bonne foi.

Or ce principe, bien que la notion semble vague, va considérablement encadrer la phase d'exécution, car il s'agit d'une règle régissant le comportement des États plus qu'une simple devise morale ${ }^{59}$. À ce titre, ce principe tend à limiter la marge d'appréciation des États. Le fait de faire des choix entre différentes possibilités dans la mise en œuvre de l'arrêt, à travers l'accord post-juridictionnel, induit la nécessité de les opérer de bonne foi pour pouvoir mettre fin au litige. En effet, la bonne foi va être «le fondement de la confiance mutuelle des États et de leur coopération ${ }^{60} »$. Ce principe va donc être indispensable dans l'exécution des décisions de la $\mathrm{CIJ}$, surtout en matière de délimitation maritime étant donné que, touchant à la souveraineté des États, cette étape postjuridictionnelle nécessite d'être empreinte de consensualisme et de ce fait, va rendre indispensable la collaboration entre les États parties au différend.

De ce principe va découler différentes obligations dans la phase d'exécution. Ainsi, l'obligation de bonne foi va souvent être liée à la notion de «raisonnable», et notamment de "délai raisonnable» dans le cadre de l'exécution, se rapportant «à ce qui est convenable, suffisant, acceptable $^{61}$ ». Par exemple, dans l'affaire de la délimitation terrestre et maritime entre le Cameroun et le Nigeria ${ }^{62}$, le fait que l'exécution de l'arrêt n'ait eu lieu que six ans après son prononcé est considéré comme

57. Cependant, il est utile de préciser que la simple référence à la Charte des Nations unies ne suffit pas à justifier de l'obligation d'exécution des arrêts de la Cour étant donné qu'il n'y a pas nécessairement concordance entre membres de l'ONU et parties au statut, du moins en théorie.

58. Voir le débat sur la valeur de l'obligation de bonne foi dans la thèse d'Affef Ben Mansour, op. cit., p. 113 et suiv., $\$ 152$.

59. Voir Affef Ben Mansour, op. cit., p. 117, $\$ 162$.

60. Ibid., p. 113, \$153.

61. Ibid., p. 120, $\$ 166$.

62. CIJ, Frontière terrestre et maritime entre le Cameroun et le Nigeria, op. cit. 
inacceptable, notamment parce qu'elle a été entravée par la réticence du Nigeria à satisfaire à ses obligations.

Cependant, l'obligation de bonne foi s'apprécie in concreto, c'està-dire qu'il faut prendre en compte la nature et la difficulté des mesures à mettre en œuvre pour exécuter l'arrêt. Cela est d'autant plus nécessaire qu'en matière de délimitation maritime, ce n'est pas chose aisée. C'est pourquoi, à l'inverse de l'affaire susvisée, Affef Ben Mansour rapporte que le délai de plus de deux ans entre le prononcé de l'arrêt en 1993 dans l'affaire de la délimitation dans la région située entre le Groenland et Jan Mayen ${ }^{63}$ et le premier accord post-jugement datant de 1995 ne fut pas considéré comme déraisonnable, car les États devaient coopérer et résoudre certaines questions qui n'avaient pas été abordées par la CIJ. De même, en cas de demande en interprétation de l'arrêt, comme dans l'affaire du plateau continental entre la Tunisie et la Libye ${ }^{64}$, il fut considéré normal que l'exécution soit retardée, tant que ce type de recours était effectué de bonne foi et ne consistait pas en des manœuvres dilatoires ${ }^{65}$.

Cette interprétation permet donc encore une fois de limiter la liberté des États qui ne pourront, de ce fait, pas user de manœuvres dilatoires pour retarder les effets du jugement. Cela permet en outre d'assurer une bonne exécution sur plusieurs plans et par conséquent de garantir la fin du litige entre les États parties au différend, ce qui est l'objectif de l'action de la CIJ.

Ainsi, « le principe de bonne foi est un catalyseur qui permet de pallier les éventuels abus et lenteurs dans la réalisation de l'obligation de mettre en œuvre un arrêt ou une décision d'une juridiction internationale ${ }^{66}$ ». En fin de compte, si le consentement de l'État et le respect de leur volonté par la CIJ va expliquer la rareté des cas d'inexécution - voire leur absence en matière de délimitation maritime -, il est cependant nécessaire qu'un certain encadrement guide l'exécution des décisions de la Cour. On peut donc affirmer que si la volonté des États parties au différend encadre le processus décisionnel de la CIJ, l'encadrement de la volonté de ces mêmes États permet quant à elle une exécution efficace de la décision. Mais la seule force obligatoire des arrêts, même doublée de l'obligation

63. CIJ, Délimitation maritime dans la région située entre le Groenland et Jan Mayen, recueil, 1993.

64. CIJ, Plateau continental (Tunisie c. Jamahiriya arabe libyenne), op. cit.

65. Pour plus de précisions sur ces exemples, voir Affef Ben Mansour, op. cit., p. 125, $\$ 174$.

66. Ibid., p. $125, \$ 175$, reprenant une métaphore de M. Virally. 
de bonne foi ne suffit pas toujours à assurer la bonne exécution des décisions de la CIJ, comme ce fut le cas de l'affaire entre le Cameroun et le Nigeria ${ }^{67}$. C'est pourquoi, en plus de cette limitation dans la liberté laissée aux États dans la phase de mise en ouvre, il est apparu de plus en plus nécessaire de permettre à la Cour de disposer d'autres moyens afin d'éviter ce genre de désagrément qui pourraient mettre en péril la stabilité des relations internationales.

\section{Une tendance au renforcement de l'encadrement de la phase d'exécution}

C'est dans un souci d'efficacité de la mise en œuvre de ses décisions que l'on observe une tendance de la CIJ à aller, dès le jugement, audelà des demandes des parties au différend et de formuler des solutions très précises, dont il est difficile de s'éloigner lors de l'application de l'arrêt ; limitant ainsi d'autant plus la marge de manœuvre des parties au différend et par la même, le risque de ne pas mettre effectivement fin au litige. Mais, bien que cette stratégie de la Cour soit déjà une avancée, serait-il opportun d'aller encore plus loin et de lui octroyer un réel pouvoir de contrainte dans son encadrement de l'exécution, à l'instar de certaines pressions qui peuvent être exercées sur les États lors de la phase post-juridictionnelle?

\subsection{L'émancipation de la ClJ par rapport aux obligations issues du compromis}

Il est observé que les juges de la CIJ semblent « s'affranchir timidement de leur position traditionnelle et accepter de préciser les modalités de l'exécution de [leurs] arrêts $^{68} »$. Ce constat s'explique par le fait que l'obligation d'exécuter et de le faire de bonne foi ne suffit pas toujours à garantir la fin d'un conflit entre États, surtout quand celui-ci touche à leur souveraineté. Néanmoins, en principe, la Cour ne dispose que de peu de pouvoirs dans la phase juridictionnelle, étant donné qu'elle doit strictement respecter les limites de la mission qui lui a été confiée par les parties au différend dans le compromis. Concernant la phase postjuridictionnelle, ce n'était guère mieux. Mais si pendant longtemps la $\mathrm{CIJ}$ a eu tendance à se déclarer incompétente et à refuser de se prononcer sur les modalités d'exécution de ses arrêts, il est observé depuis quelques temps que les juridictions internationales ont tendance à « s'émanciper

67. CIJ, Frontière terrestre et maritime entre le Cameroun et le Nigeria, op. cit.

68. Ibid., p. $127, \$ 180$. 
de la limitation sur la précision des modalités de la mise en œuvre de leurs arrêts ${ }^{69} »$. Bien que ce phénomène soit plus flagrant chez les juridictions régionales, souvent dotées de mécanismes d'application de leurs décisions et d'organes de contrôle de l'exécution, la CIJ va également être influencée par cette manière de procéder permettant de parvenir à une exécution efficace et satisfaisante. Cet impératif d'efficacité est d'autant plus important que le domaine des délimitations maritimes est sensible et touche directement à la stabilité des relations interétatiques. Il faut donc que l'arrêt soit exécuté promptement et efficacement par les États parties au différend afin de véritablement mettre fin au litige.

La clarté du contenu de la décision est alors très importante car elle va permettre d'éviter les recours en interprétation, consistant le plus souvent en des procédures dilatoires freinant l'exécution de l'arrêt. D'autre part, un dispositif exposant clairement les modalités de mise en œuvre entraîne une mise en œuvre plus rapide puisque les États n'ont pas à déterminer les obligations découlant de l'arrêt lors de l'accord postjuridictionnel. Il ne s'agit pas d'injonction, l'étendue de l'intervention de la Cour étant limitée par la barrière de la sacro-sainte souveraineté étatique rendant difficile toute exécution forcée, mais d'orientations prononcées avant même la phase post-juridictionnelle, afin de prévenir les potentielles difficultés d'exécution.

Cependant, les précisions apportées par la CIJ vont être relativement indirectes et implicites. En effet, la Cour, dans sa politique consensuelle, ne souhaite pas que les États se sentent atteints dans leur souveraineté, ce qui pourrait avoir pour conséquence néfaste un rejet de la décision. Le problème en matière de délimitation maritime, consiste en ce que lorsque le compromis demande uniquement à la Cour de déterminer les principes applicables à la délimitation, il paraît difficile de préciser d'avantage les modalités d'exécution. Pourtant, dans cette volonté de permettre une exécution efficace, la Cour va parfois aller au-delà de ses prérogatives et s'imposer en guide afin de rendre le prononcé clair pour les parties et donc plus facilement exécutable. En matière de délimitation maritime par exemple, dans l'affaire de la délimitation maritime dans la région située entre le Groenland et Jan Mayen ${ }^{70}$, la Cour va donner les coordonnées nécessaires au tracé en précisant les longitudes et les

69. Ibid., p. 126, \$176.

70. CIJ, Délimitation maritime dans la région située entre le Groenland et Jan Mayen, op. cit. 
latitudes ${ }^{71}$. Parfois, lorsqu'il lui a seulement été demandé de donner les règles de droit et principes applicables à la délimitation, elle va en plus préciser les circonstances qu'il faudrait prendre en compte pour obtenir un résultat équitable ${ }^{72}$. Cette tendance s'observe également dans d'autres domaines, comme ce fut le cas lors de l'affaire de 2005 opposant la république démocratique du Congo à l'Ouganda où la Cour avait décidé de se déclarer toujours saisie tant que la phase de réparation n'avait pas été épuisée ${ }^{73}$. Bien que ces méthodes pratiques ne lient pas véritablement les États puisque l'exécution relève d'une obligation de résultat et non de moyen, elles permettent tout de même de les orienter et de prévenir toutes difficultés d'application. Néanmoins, dans certaines affaires, le prononcé de l'arrêt et les méthodes d'exécution n'ont pas pu empêcher des requêtes en interprétation ${ }^{74}$.

Ainsi, l'obligation de mise en œuvre des arrêts est moins flexible qu'il n'y paraît à première vue. Si la liberté des États dans l'exécution est justifiée par la souveraineté, elle est néanmoins limitée par l'exigence de respecter au mieux les délimitations maritimes établies par la CIJ. Or la satisfaction de cette obligation de résultat peut être favorisée si la Cour oriente - ou du moins précise - ses décisions. Cela permet de faciliter le travail des États sans pour autant toucher à leur souveraineté. Et «en définitive, ce rôle que la Cour est susceptible de s'attribuer en certaines occasions constitue, en quelque sorte, le versant préventif de l'exécution forcée. En effet, en assistant les parties, la Cour prend les mesures susceptibles d'empêcher la phase post-juridictionnelle de dégénérer en une violation de l'obligation d'exécution, situation qui nécessiterait la mise en place d'une procédure d'exécution forcée ${ }^{75}$ ». Mais si la CIJ n’a jamais eu recours à l'exécution forcée, la tendance observée pose néanmoins la question de savoir si l'on n'assisterait pas aux prémices de la mise en place d'une obligation de moyen dans l'exécution de ses arrêts.

71. Ce fut le cas notamment dans l'affaire de la délimitation maritime dans la région située entre le Groenland et Jan Mayen (op. cit., p. 47-48, \$ 93), ainsi que dans l'affaire de la délimitation maritime dans la région du Golfe du Maine (op. cit., p. 345 $\$ 243)$.

72. Voir notamment: CIJ, Plateau continental (Jamahiriya arabe libyenne c. Malte), op. cit., p. $57, \$ 79$.

73. CIJ, Activités armées sur le territoire du Congo (République démocratique du Congo c. Ouganda), recueil, 2005, p. 117, point 6 et p. 118, point 14 .

74. CIJ, Plateau continental (Jamahiriya arabe libyenne c. Malte), recueil, 1985, p. 79, $\$ 133 \mathrm{C}$.

75. Aïda Azar, op. cit., p. 128-129, \$212. 
À ce sujet, le rapport de la sixième commission de l'assemblée générale des Nations unies du 29 octobre 2010 (AG/J/3403) s'arrête plus précisément sur l'affaire Avena et autres ressortissants mexicains opposant le Mexique aux États-Unis ${ }^{76}$, à l'occasion de laquelle ces derniers avaient refusé de se conformer à l'arrêt, sous prétexte de difficultés de mise en œuvre dans leur ordre juridique interne, du fait de l'absence d'obligation contraignante issue de l'arrêt. La commission souligne également la similarité du cas avec celui du Nigeria dans l'affaire de la délimitation maritime l'opposant au Cameroun ${ }^{77}$.

Il est vrai que la seule obligation des États durant l'exécution étant, du moins en principe, une obligation de résultat, leur liberté est très largement étendue et cette liberté peut donner lieu à des manœuvres freinant, voire empêchant, la bonne exécution des décisions de la Cour. Se pose alors la question de savoir s'il ne serait pas opportun d'instaurer, dans le cadre de la phase post-juridictionnelle, des obligations de moyens permettant a minima d'encadrer les États parties au litige dans le processus d'exécution. Il est indéniable que la Cour exerce un important travail en amont pour assurer l'acceptation et le respect de sa décision, et qu'elle va parfois jusqu'à véritablement limiter la marge de manœuvre des États dans le prononcé. Il n'existe cependant pas de contrainte réelle pesant sur les États pour qu'ils effectuent un processus d'exécution permettant de parvenir au règlement du conflit; l'intervention autoritaire du Conseil de sécurité étant peu conseillée car elle risque d'entraîner le rejet catégorique de la décision.

Or c'est véritablement la notion d'état de droit qui est en jeu ici. En effet, comme le souligne le juge Owada, "l'exécution des arrêts de la CIJ, qui est un pan notable de l'action de la Cour en matière de règlement pacifique des différends, constitue la pierre angulaire de la primauté du droit au sein de la communauté internationale». Il poursuit en expliquant que « la question du respect et de l'exécution des décisions rendues par la $\mathrm{CIJ}$ est très importante pour la consolidation de l'état de droit dans le droit international ${ }^{78} »$. À ce titre, le secrétaire général de l'ONU a décidé de créer un groupe sur la coordination pour l'état de droit. Cette exigence de respect de l'exécution des décisions de la Cour est d'autant plus importante que le conflit entre l'ordre juridique international et l'ordre juridique interne risque de s'intensifier,

76. CIJ, Avena et autres ressortissants mexicains (Mexique c. États-Unis d'Amérique), recueil, 2004.

77. CIJ, Frontière terrestre et maritime entre le Cameroun et le Nigeria, op. cit.

78. Observation du juge Owada (déclaration citée supra). 
du fait de leurs interactions croissantes et de l'intégration du premier dans le second, notamment dans des domaines, à l'instar de celui des délimitations maritimes, touchant à la souveraineté des États.

Mais si imposer une obligation de moyen serait une technique efficace pour assurer une bonne exécution de ses arrêts sans pour autant constituer une ingérence dans les affaires internes des États en cause, il semble en revanche impératif de préciser que cette dernière ne devra pas supplanter l'obligation de résultat mise à la charge des États dans l'exécution. Dans le cas contraire, les parties n'auraient plus cette obligation de parvenir au résultat escompté par tout recours à la CIJ : le règlement du différend. Toutefois, si les États respectent un certain processus d'exécution dans le cadre de l'obligation de moyen, la fin du litige par l'exécution de la décision serait assurée. À ce propos, la Cour a d'ores et déjà expliqué que l'obligation de négocier de bonne foi un désarmement nucléaire contenue à l'article VI du traité de nonprolifération des armes nucléaires consiste en une « double obligation de négocier et de conclure ${ }^{79} »$; il s'agit donc d'une exigence de moyen et de résultat en même temps. Cette attente pourrait tout à fait être transposée à l'obligation d'exécuter les arrêts de la Cour.

En attendant l'institutionnalisation d'un tel bouleversement dans les obligations mises à la charge des parties dans la phase post-juridictionnelle, il est observé dans la pratique l'existence de véritables contraintes qui vont bien au-delà de l'ébauche d'encadrement effectué par la CIJ, exercée notamment par la communauté internationale lorsque la mauvaise volonté des États dans l'exécution des arrêts de la Cour risque de menacer la paix et la sécurité internationale. Ce mode de fonctionnement qui a su faire preuve d'efficacité est-il pour autant transposable à la Cour?

\subsection{L'opportunité d'octroyer un véritable pouvoir de contrainte à la CIJ : une question encore en débat}

Pour aller plus loin vers le risque zéro en matière d'inexécution et ainsi assurer l'effectivité et l'efficacité de la justice internationale, il serait peut-être opportun de réduire encore la place de la volonté des parties au différend lors de la phase post-juridictionnelle en agissant plus par la contrainte que par un contrôle minimum, voire uniquement par l'influence. Mais cela entraînerait des difficultés d'acceptation de la part des parties, ces dernières recourant à la CIJ justement parce qu'elles ont

79. CIJ, Licéité de la menace ou de l'emploi d'armes nucléaires, avis consultatif, recueil, 1996, p. 41-42, \$99-100. 
cette marge de manœuvre. C'est pourquoi le consentement doit être préservé.

Mais cette "contrainte» sur la mise en œuvre des décisions de la Cour existe déjà, de fait. Elle se trouve parfois en dehors des organes de l'ONU. En effet, lors de l'exécution des décisions de la CIJ notamment en matière de délimitation maritime qui nécessite des modalités d'exécution relativement compliquées du fait de la nature du territoire sur lequel il faut opérer - les États peuvent décider de recourir à l'assistance non pas de la CIJ, mais à celle d'autres organisations internationales. Ce fut le cas dans l'affaire du différend frontalier terrestre, insulaire et maritime du 11 septembre 1992 opposant le Salvador au Honduras, durant l'exécution de laquelle les États ont eu recours à l'Organisation des États américains afin d'appliquer la décision ${ }^{80}$ (notamment pour la frontière terrestre).

Mais il est également possible que ce soit la société internationale ellemême qui réagisse au blocage de l'exécution. Dans cette configuration aussi, le consentement des États en cause est indispensable, du moins en théorie. En effet, la différence fondamentale est qu'ici, la pression qu'exerce la communauté internationale ne laissera pas vraiment le choix d'accepter ou non cette assistance, malgré un différend dont l'objet touche à la souveraineté même des États. La seule illustration de ce cas de figure en droit des délimitations maritimes est celle de l'affaire de la frontière terrestre et maritime entre le Cameroun et le Nigeria ${ }^{81}$. Ce fut également un des rares cas de "mauvaise exécution » en la matière; dans le sens où la durée du processus d'application de la décision montre une certaine mauvaise volonté de faire cesser le conflit. Pour le reste, l'ONU n'a pas eu l'occasion de réagir puisque soit l'arrêt était bien exécuté, soit les difficultés d'application ne menaçaient pas le maintien de la paix.

Dans ladite affaire, l'objet principal de la délimitation (maritime et terrestre) était la souveraineté sur la péninsule de Bakassi ; territoire riche en gisements de pétrole, de gaz et en ressources halieutiques. Étant donné le comportement hostile du Nigeria durant la procédure, le Secrétaire général des Nations unies avait, avant même le prononcé de l'arrêt, invité les présidents respectifs des États parties au différend à se réunir afin d'obtenir un accord de principe sur l'application de la décision

80. CIJ, Différend frontalier terrestre, insulaire et maritime (El Salvador c. Honduras, Nicaragua (intervenant)), recueil, 1992.

81. CIJ, Frontière terrestre et maritime entre le Cameroun et le Nigeria, op. cit. 
de la Cour ${ }^{82}$ mais ce n'eut pas l'effet escompté. C'est véritablement le prononcé de l'arrêt par la CIJ, le 10 octobre 2002, qui fit l'effet d'un coup de tonnerre, surtout du côté nigérian qui réagit de manière très virulente ${ }^{83}$. Ainsi, une seconde rencontre fut mise en place par le secrétaire général des Nations unies à Genève le 15 novembre 2002, qui donna lieu à la création d'une commission mixte Cameroun-Nigeria, sous la présidence du représentant spécial du secrétaire général des Nations unies. Cette commission mixte, après vingt-deux échecs, a enfin réussi à amener le Nigeria à accepter la décision de la Cour en février 2003. Mais le manque de volonté du Nigeria a ralenti le processus jusqu'à ce qu'enfin, le 12 juin 2006, soit signé à Greentree, près de New York, l'accord post-jugement qui marqua le début de l'application effective de l'arrêt.

Après le rôle joué par les Nations unies, l'intervention des États tiers au différend dans l'exécution de l'arrêt peut se traduire par des aides financières. En effet, après la mise en place d'un fond d'affectation spécial par les Nations unies, les États-Unis, l’Union européenne et d'autres grandes puissances contribuèrent à favoriser la fin du conflit et l'exécution de la décision de la Cour $^{84}$.

Ainsi, l'intervention de tiers sans que les États parties au différend n'aient sollicité leur concours est un moyen plutôt efficace d'assurer l'application des décisions de la CIJ, même dans des matières à dimension fortement émotionnelles comme les délimitations maritimes. Bien que le consentement de l'État soit indispensable, il est également indéniable que la pression exercée par la communauté internationale a au moins

82. Mahmoud Mohamed Salah, «La Commission mixte Cameroun/Nigeria, un mécanisme original de règlement des différends interétatiques", AFDI, 2005, p. 164.

83. Pierre d'Argent, «Des frontières et des peuples : l'affaire de la frontière terrestre et maritime entre le Cameroun et le Nigeria (arrêt sur le fond)», AFDI, 2002, p. 282 : "In the instant case, for purely political reasons, the Court, headed by a french President, upheld a legal position which is contrary to all known laws and conventions, thus legitimising and promoting the interest of former colonial powers at our expense. The french President of the Court and the English and German judges should have disqualified themselves since the countries which they represent are, in essence, parties to the action or have substantial stakes. These Judges, as citizens of the colonial powers whose action had come under scrutiny, have acted judges in their own cause and thereby rendered their judgement virtually null and void. Nigeria does not accept that a Protectorate Treaty made without jurisdiction should take precedence over community's title rights and ownership existing from time immemorial. Great Britain could not have given to Germany what it did not have. For a stronger reason, what Germany did not have could not have been transferred to Cameroon. "

84. Voir Géraldine Giraudeau, op. cit., p. 120. 
influencé les parties dans leur volonté de mettre fin au différend, voire a permis la résolution de ce différend.

Certains estiment d'ailleurs que :

[...] globalement, la mauvaise volonté des États ne semble pas devoir être retenue pour expliquer certaines difficultés d'exécution des décisions de la CIJ. Les cas observés invitent plutôt à s'interroger sur l'adéquation du procès au règlement de certains différends. Si l'utilité du règlement juridictionnel n'est pas mise en cause, la question des suites met en évidence l'insuffisance de l'articulation entre la procédure juridictionnelle et les autres éléments du processus de règlement des différends. Le besoin d'une aide extérieure manifestée par les parties à travers la saisine du juge n'est pas nécessairement satisfait par le prononcé de la sentence et l'exécution assistée peut améliorer substantiellement l'efficacité de la justice internationale ${ }^{85}$.

D'où l'idée d'octroyer à la Cour un pouvoir similaire à celui qu'exerce la communauté internationale. Cependant, les États ne sont pas encore prêts à abandonner une large part de l'exercice de leur souveraineté au profit de l'action de la Cour, c'est la raison pour laquelle la question de l'extension des pouvoirs de la CIJ fait actuellement débat.

Pourtant, selon l'article 38 de son statut, la CIJ a pour mission de régler les différends qui lui sont soumis conformément au droit international. Étant un des six principaux organes de l'ONU et le principal organe judiciaire, la Cour doit satisfaire aux buts des Nations unies, dont celui du maintien de la paix et de la sécurité internationale. En ce sens, la compétence limitée conférée à la Cour dans la phase postjuridictionnelle semble incompatible, du moins contradictoire, avec la mission de l'ONU. En effet, la Cour est investie d'une mission pacificatrice dans sa fonction judiciaire mais, comme nous l'avons vu, ses moyens d'action sur l'exécution sont insuffisants. Afin d'assurer parfaitement cette fonction, il faudrait lui reconnaître « une compétence implicite dans l'exécution des décisions dans la mesure où le refus d'exécuter peut, dans certaines hypothèses, menacer la paix internationale ${ }^{86}$ " et ce, en vertu d'une interprétation extensive de ses compétences issues du compromis.

D'ailleurs, une telle interprétation semble possible puisque certaines cours, notamment régionales, ont d'ores et déjà eu recours à ce type de raisonnement. C'est le cas par exemple de la Cour interaméricaine des droits de l'homme (CIADH) qui est réputée pour ses interprétations

85. Philippe Weckel, «Les suites des décisions de la Cour internationale de Justice», AFDI, 1996, p. 440.

86. Aïda Azar, op. cit., p. 201, \$325. 
extensives et parfois même audacieuses ${ }^{87}$. Dans le même sens, la CIJ a déjà eu recours à la théorie des compétences implicites, notamment dans l'affaire des essais nucléaires où elle s'octroie un pouvoir lui permettant de prendre toute mesure voulue pour que l'exercice de sa fonction soit efficace $^{88}$. Il serait donc imaginable que la Cour étende ses pouvoirs au-delà de la phase strictement juridictionnelle et puisse agir jusqu'à ce que les États parties au différend acceptent de mettre en œuvre le jugement, exerçant ainsi une certaine forme de contrainte.

Étant donné que cette compétence n'est reconnue par aucun texte, Aïda Azar propose que la Cour interprète le compromis de manière extensive, car encore une fois, le consentement des États est indispensable pour s'octroyer une telle extension de pouvoir. Ainsi, la justification consisterait à dire que, lorsque les parties prévoient dans le compromis la conclusion d'un accord post-jugement afin de déterminer le détail de l'exécution - élément indispensable spécifiquement en matière de délimitation maritime -, la Cour pourrait se reconnaître le pouvoir de négocier cet accord avec les États parties au différend puisque la compétence de la CIJ a été associée à cet accord. Cela permettrait en outre de limiter l'affaiblissement de la force obligatoire de ses arrêts puisque la Cour aurait un certain contrôle sur la marge de négociation de l'arrêt. De même, lorsque les parties prévoient dans le compromis le recours à la CIJ en cas de désaccord sur les suites données à l'arrêt, comme ce fut le cas dans l'affaire du plateau continental ${ }^{89}$ (Tunisie c. Libye), la Cour, au lieu d'attendre qu'une ou les deux parties la saisisse de nouveau, pourrait se reconnaître compétente pour se saisir d'office, si elle observe un blocage dans la phase d'exécution, vu que les États avaient prévu ce recours dans la phase pré-juridictionnelle.

Cependant, il ne faut pas perdre de vue que si la Cour est saisie, notamment dans des contentieux relativement difficiles comme celui de la délimitation maritime, c'est parce que les États savent qu'ils vont avoir une certaine maîtrise du processus. Or cette extension de compétence réduirait leur marge de liberté; ce qui pourrait nuire au nombre de saisines de la Cour. C'est pourquoi, au lieu d'opérer une interprétation extensive de sa compétence, il est également imaginable

87. En effet, pour exemple, dans le cadre de la réparation, la CIADH a développé la théorie du dommage au projet de vie lors de l'arrêt Loayza Tamayo c. Pérou du 27 novembre 1998, recueil, p. 39, \$147.

88. CIJ, Licéité de la menace ou de l’emploi d'armes nucléaires, op. cit., p. 259 et 463; exemple cité dans Aïda Azar, op. cit., p. 202, \$326.

89. CIJ, Plateau continental (Tunisie c. Jamahiriya arabe libyenne), op. cit., p. 40, $\$ 31$. 
de se tourner vers la piste de l'amendement du statut de la Cour, possible en vertu des articles 69 et 70. Car si la théorie des compétences implicites ne pose pas de problème à certaines juridictions régionales, c'est parce qu'elles ont une compétence obligatoire à l'égard des États ${ }^{90}$; les parties n'ont donc pas le choix d'y recourir lorsque le litige rentre dans leur compétence ratione materiae, ce qui n'est pas le cas de la CIJ. Ainsi, après modification du statut, il serait envisageable d'octroyer à la Cour la possibilité d'être de nouveau saisie par une des parties en cas d'inexécution dans un délais qu'elle aurait fixé au moment du prononcé de l'arrêt. Elle pourrait donc par la suite contraindre l'État débiteur à appliquer l'arrêt ou trouver un nouveau compromis acceptable par les parties. Cette démarche demanderait une modification de grande ampleur du statut de la Cour car elle lui permettrait d'être l'organe d'appel de ses propres décisions.

Enfin, il pourrait être donné à la Cour la possibilité de demander aux parties de l'informer sur le déroulé des suites de l'arrêt. En cas d'inexécution, elle pourrait saisir elle-même le conseil de sécurité, alors qu'actuellement, seules les parties au différend peuvent le faire ${ }^{91}$.

Pourtant, si les possibilités de renforcement des pouvoirs de la CIJ sur la phase post-juridictionnelle sont relativement nombreuses, les États ne semblent pas encore prêts à céder une partie de leur souveraineté dès lors qu'ils saisissent la Cour. Cependant, l'influence que les juridictions exercent les unes sur les autres pourrait à l'avenir venir modifier le mode de fonctionnement de la CIJ s'il est avéré que la «contrainte » exercée lors de l'exécution de l'arrêt est proportionnée et adéquate.

La CIJ exerce donc une influence certaine sur la phase d'exécution de ses arrêts, voire un véritable encadrement. Mais en réalité, n'est-ce pas ce processus de conciliation et d'encadrement combiné à la liberté des États parties au différend qui permet d'expliquer la volonté de ces derniers à exécuter la décision de la Cour ainsi que la mise en place d'un véritable processus de réconciliation et de coopération entre les États durant l'étape post-juridictionnelle? Cependant, pour des raisons tenant au respect de la toute-puissante souveraineté des États, il n'est pas encore question d'aller au-delà du simple encadrement; l'exécution

90. Comme c'est le cas de la Cour de justice de l'Union européenne. En revanche, ce n'est pas le cas de la Cour interaméricaine des droits de l'homme ou la Cour européenne des droits de l'homme.

91. Pour ces différents exemples d'extension des pouvoirs de la Cour dans la phase post-juridictionnelle, voir Aïda Azar, op. cit., p. 205-208, \$332-336. 
forcée restant une exception et n'ayant encore jamais été utilisée dans le cadre des délimitations maritimes. S'il est vrai que l'État tient encore à garder une certaine maîtrise sur la décision et ses suites, il serait néanmoins plus utile encore qu'il accepte, dans le cadre d'un projet de société et de justice internationale cohérente et efficace dans le respect des règles de droit, certaines contraintes afin d'assurer l'exécution des décisions de la CIJ dans les différents cas de figure possibles. C’est sur ce choix que s'exerce véritablement la liberté des États ${ }^{92}$.

Doctorante à l'université de Rouen, faculté de droit, sciences économiques et de gestion, membre du CUREJ (Centre universitaire rouennais d'études juridiques)

92. Je tiens à remercier Abdelwahab Biad, directeur de mon mémoire portant sur ce sujet, ainsi qu'Anne-Thida Norodom, pour leurs conseils et leurs regards critiques lors de la rédaction de cet article. 